"On ne peut nier que les Français soient les initıateurs de l'électrométallurgie du fer, mais ils ne l'appliqueront qu'après les Allemands et les Américains ".

on nous accorde l'esprit d'intuition et de synthèse, la conception claire el limpide qui fait les inventeurs, mais on nous refuse l'esprit pratique, la patience et la persévérance exigées pour l'application industrielle. On dıt aussi que nous n'avons pas confiance en nous-mêmes. Mais, que ne diton pas?

Quant à moi, je ne crois pas, je ne veux pas crorre, que nous soyons en voie de décadence industrielle, et il suffirait de l'admlrable essor de l'industrie automobile, crée et continuée par nous, pour prouver que nous sommes loujours à l'avant-garde du progrès et que nous savons nous y maintenır.

Il en sera de mẻme pour ce qui concerne l'acier électrique; je le crois et je le dis : j'ai la ferme espérance que ce sera encore le coq gaulois qui chantera au sommel de l'édifice elevé prerre à pierre par les Français et achevé par eux.

G. GIN.

\section{Quelques Projets Gigantesques}

La presse technique en France et à l'étranger a récemment publié les informations suivantes qu'à notre tour nous ne pouvons manquer de reproduire sous peine de paraître ignorant des grands projets.

Il s'agir d'abord d'un projet dû à $\mathrm{M}$. Guarini, professeur à l'Ecole des Arts et Métiers de Lima, qui propose l'utilisation industrielle des eaux du lac Titicaca. S'étendant sur une superficie de 6630 kilomètres carrés, sous une profondeur moyenne de 20 mètres, ce lac constitue un réservoir de 132600 ooo mètres cubes. L'auteur de ce gigantesque projet indique la possibilité de canaliser les eaux de ce réservoir naturel vers la côte du Pacifique, assez proche, en suivant les pentes rapides de la Cordillière des Andes. Le lac étant à 3800 mètres d'altitude, on pourrait, en dérivant 100 mètres cubes par seconde, produire au moins 2 millions de chevaux pratiquement (?) utilisables sur l'arbre des turbines.

Les moyens proposés par M. Guarini pour opérer cette dérivation sont, en principe, très simples. Le lac étant à 250 mètres en dessous du point le plus bas de la chaîne de montagnes quile sépare de la côte, on a le choix, dit-il, entre un tunnel perçant la montagne en ligne droite et le dispositif suivant qui serait plus élégant, et peut-être moins coûteux : des pompes actionnées par l'énergie des chutes qui seraient établies à la suite les unes des autres, entre le sommet de la montagne et la côte, relèveraient l'eau du lac à 250 mètres et, de là, cette eau s'écoulerait vers le Pacifique en traversant une série d'usines échelonnées sur une hauteur totale de plus de 3000 mètres; au rendement près des pompes et des conduites, on récupérerait ainsi l'énergie dépensée à l'élévation. Après a voir traversé les dernières turbines, l'eau servirait à l'irrigation des cuitures de la côte.

D'après un avant-projet sommaire, le coût des installations serait d'un ordre de grandeur qui ne dépasse pas l'inagination, puisqu'avec 200 millions, paraît-il, on pourrait peut-être en voir le bout! Cela mettrait les frais de premier établissement du cheval à 100 francs. Nous connaissons plus d'un auteur de projets qui, dans nos Alpes, a fait ressortir les frais d'installation du cheval a moins de 100 francs surl'arbre des turbines. Ce prix n'est donc pas exagéré dans le sens du bon marché qu'on pourrait s'attendre à voir ressortir de ces grandioses conceptions auxquelles nos confrères du Nouveau-Monde nous ont quelque peu habitués. Mais ce que nous ne chercherons même pas à mettre en comparaison avec ce que nous connaissons de nos pauvres petites installations alpestres, ce sont les éléments d'appréciation des puissances aménageables et les moyens de construction. En Amérique, comme on le sait, tout est grand, et nous aurions mauvaise grâce, nous petits alpins, à discuter des projets de géants.

Cette énorme quantilé d'énergie pourrait être utilisée à la traction électrique des chemins de fer, à l'électrochimie et à l'électrométallurgie, aux services des mines du Pérou et de l'agriculture, rotamment au labourage électrique, à l'éclairagje des localités et enfin à la fabrication des nitrates pour concurrencer les gisements naturels du Chili.

Que le Niagara se tienne bien... ou il est enfoncé !

Mais il n'y a pas que les Américains pour faire grandement les choses; nous aussi, en Europe, sans vouloir leur disputer le record des chutes monstres, nous pouvons néanmoins leur opposer des forces hydrauliques respectables.

Le projet suivant, dû à $M$. Von Dowat, permettrait de créer, pas bien loin d'ici, 300000 chevaux, tout simplement. Cette fois, il nous est facile d'y aller yoir, c'est en Bavière, Et nous qui mettions l'Allemagne au rang des pays peu fortunés sous le rapport de la houille blanche !...

Voici, dans ses grandes lignes, l'économie du projet"en ques. tion: sur l'Isar, à moitié chemin entre Wallgan et Vorderiss, on construirait un barrage - de dimensions telles que jamais circulaire ministérielle n'en a encore prévu chez nous - et qui retoulerait les eaux de la rivière jusque dans un petit lac alpestre de 650 miètres de large et 6 kilomètres de long. Ce serait là un premier réservoir emmagasinant 65 millions de mètres cubes. De ce lac, l'eau serait dirigée au moyen d'un tunnel de 3800 mètres, dans le lac Walchen, situé à 52 mètres plus bas. La réalisation de cette différence de niveau dans une chute hydraulique donnerait dabord 20000 chevaux. Par une seconde galerie, les eaux du Walchen seraient envoyées dans le lac Kochel en franchissant une seconde chute de 203 mètres où l'on recueillerait 80000 chevaux.

Ce lac reçoit aussi les eaux du Loisach, torrent à débit très variable. On établirait alors un barrage sur ce torrent, pour créer un lac artificiel de Tscheulohe à Oberau et capable de contenir 100 millions de mètres cubes. Ce réservoir pourrait fournir un débit de 63 mètres cubes à la seconde qui, joints aux 23 mètres cubes fournis par l'Isar et ses affluents, donnerait un débit total de 86 mètres cubes par seconde. Ce volume pourrait être utilisé sous une nouvelle différence de niveau de 300 mètres permettant d'obtenir 200000 chevaux dans cette troisième chute.

Au total, on aurait ainsi 300000 chevaux disponibles.

Cela ne vaut pas le Titicaca, mais enfin c'est déjà une puissance capable de satisfaire à quelques-unes des demandes d'énergie que la paisible industrie de notre vieille Europe réclame pour se maintenir au niveau de celle du Nouveau Monde.

Il paraît que les Bavarois discutent ferme, non pas tant sur la possibilité de mener à bien cette entreprise, du reste parfaitement exécutable sous le rapport technique, que sur le point de savoir si ces barrages, ces usines avec leurs tuyaux monstres et leurs réseaux de fils et de poteaux, ne nuiront point aux charmes du paysage, ne "matérialiseront "pas trop cette idéale région des Alpes, célèbre par le pittoresque de ses sites, aussi bien que par les fêtes artistiques auxquels ils servent de cadre? On se demande si les chutards et les électriciens n'éloigneront pas a jamais les poètes et les musiciens de Bayreuth !...

Il s'agit ensuite du transport électrique de l'énergie des chutes du Zambèze, aux Victoria Falls, jusqu'aux mines du Rand, dans le Transval: la distance est de 1200 kilomètres!

D'après certaines estimations, la puissance des chutes du Zambèze aux Victoria Falls serait d'environ 500 ooo chevaux, pour une chute utile de 100 mètres. L'Afrique, on le voit, rivalise avec l'Amérique. Comme d'une part cette puissance ne parâ̂t pas immédiatement utilisable sur place - et pour cause - que d'autre part le charbon coûte très cher au Transvaal, M. Fox, directeur de la British South African Company, a étudié le projet peu banal de transporter une partie de cette énergie jusqu'aux mines du Rand qui absorbent actuellement I 50 ooo chevaux, paraît-il.

M. Fox adopte le système Thury à courant continu-série avec une tension de roo ooo volts qu'il estime ne pas devoir présenter plus de difficultés d'isolement que le courant triphasé 
à 60000 volts employé à Guanajuato, au Mexique. Pour une puissance transportée de 20000 chevaux, et en employant des pylones en fer et des cables en aluminium établis pour une perte de 33 pour 100 , la ligne coûterait 50 millions. En comp. tant un intérêt de 5 pour roo pour rémunérer le capital engagé et une dépense annuelle estimée à 5 pour $100 \mathrm{du}$ dit capital pour les frais d'entretien de la ligne, le coût du transport d'un kilowatt-heure serait de ofr. 057. M. Fox estime que les frais d'aménagement des chutes, ainsi que ceux d'établissement des stations de transformation, reviendraient à ofr. o 35 par kilowatt-heure de sorte que le prix d'un kilowatt-heure transporté au Rand reviendrait a ofr. o 705. Pour une puissance transportée plus grande, le prix de revient descendrait encore plus bas, car, pour la ligne, le prix d'établissement des pylônes ainsi que les frais d'entretien ne croissent pas proportionnellement avec l'énergie transmise.

Voilà certes un grandissime projet. Chez nous on parla voici quatre ans de transporter électriquement l'énergie d'une partie des chutes de la Savoie et du Dauphiné a Paris : la distance est tout au plus de 600 kilomètres. Comme on le voit, le transport électrique de l'énergie fait du chemin.... sur le papier. Loin de nous cependant la pensée de vouloir dire qu'il ne progresse pas réellement et qu'il se limitera pour toujours au rayon de 200 kilomètres aujourd'hui atteint entre Moutiers et Lyon par la Société Grenobloise de Force et Lumière. Nous n'avons point le don de prescience et nous ne saurions dire par quels moyens cela se fera ni à quelle date, mais les progrès étonnamments rapides de cette merveilleuse industrie nous donnent la vision intuitive de turbines hydrauliques actionnant des machines électriques à 500 kilomètres de distance. Le calcul indique la possibilité d'un pareil tour de force; reste à savoir le temps que la pratique, qui s'acquiert en ce moment sur les lignes de 200 kilomètres, mettra pour résoudre ces mille et une difficultés, d'ordre secondaire, bien connues des industriels exploitant ces lignes, et qui les font astuellement hésiter lorsqu'il s'agit de les prolonger de 50 kilomètres.

Aménager une chute d'eau ou le cheval coutera par exemple 50 francs l'an aux bornes des génératrices, et transporter cette énergie à 500 kilomètres de là dans une région ou le prix de revient du cheval-houille noire est en moyenne 300 francs, cela paraît très séduisant, et par un calcul habile autant qu'exact l'on peut faire ressortir d'une telle affaire les plus beaux côtés. Mais ce que le calcul n'indique pas, ce sont les isolateurs brisés et les tils coupés, les courts-circuits et les pertes à la terre intempestives, les accidents causés par les orages, la malveillance ou la négligence, les frais d'entretien encore trop mal connus faute d'une expérience assez prolongée. Tout cela fait dire aux industriels spécialistes que l'on connầt bien son prix de revient au dépari de l'usine génératrice, mais qu'on ne peut pas affirmer ce qu'il sera exactement à 250 kilomètres.

Quand le calcul d'un avant-projet de transport d'énergie fait ressortir le prix de revient au bout de la ligne à peu près au même chiffre que celui donné par la vapeur dans la région visée par ce transport, on ajourne l'exécution du projet parce que les alea d'exploitation des lignes font toujours pencher la balance en faveur de la houille noire; il faut alors altendre que se manifestent de nouveaux perfectionnements qui suppriment une partie de ces alea. Le système contina-série mis en pratique avec un succès croissant par l'habile ingénieur qu'est M.Thury est évidemment de nature, par l'économie qu'il apporte dans l'établissement des lignes, à prolonger beaucoup lu portée actuelle de la transmission électrique de l'énergie et nous croyons parfaitement rationnel la création de réseaux de plus de 200 kilomètres de rayon quand ils ont pour but de transporter de la houille blanche très bon marché dans des régions oul la houille noire revient très cher.

C'est assurément le cas dans lequel serait placé le transport de l'énergie des chutes du Zambèze au Transvaal. Mais sans discuter les bases du projet de M. Fox - les moyens nous en font défaut - nous nous demandons simplement si l'on peut comme cela, du jour au lendemain, quintupler les distances franchies par les lignes de transporı d'énergie électrique?Si confiants que nous soyons dans les progrès à venir, quels que. soient les concours de circonstances qui peuvent faciliter le fonctionnement de telles installations, nous n'espérons pas les voir entrer de sitôt dans le domaine de la réalité.

De tels projets sont néanmoins très intéressants à suivre en ce sens qu'ils montrent tout ce qu'on peut attendre du génit de nos constructeurs et c'est à ce titre que nous en avons fait: mention dans ces colonnes.

En attendant que se réalisent dans l'industrie du transport électrique de l'énergie, les perfectionnements nécessaires pour permettre la mise à exécution de ces grands projets, nous allons assister au développement d'une branche de l'électrochimie qui va faire mettre en œuvre de nombreuses et puissantes chutes d'eau. Nous voulons parler de la fabrication électrochimiqué des nitrates pour l'Agriculture.

Nos lecteurs ont été tenus au courant des travaux de labora. toire et des essais industriels tentés dans cette voie, puis des appareils mis en fonctionnement régulier dans quelques usines ayant, parât-il, résolu le problème au triple point de vue électrochimique, agricole et commercial. A diverses reprises, les descriptions du principe des expériences de Mc. Dougall et Howless, des procédés Johnson, Bradley et Lovejoy, expéri. mertés par lAtmosphéric Products $\mathrm{C}^{\circ}$, des appareils Kowalski. Mosciski, de Mitchell et Parks, de Marquardt et Viertel, de E. Werner, de Pauling, etc., ont parues dans nos colonnes. Nous avons également décrit les expériences et les procédés Franck et Carro pour la fabrication des cyanamides. En dernier lieu, nous indiquions le succès remarquable obtenu par M M. Birkeland et Eyde, dans leurs nouveaux fours à synthèse industrielle de l'acide nitrique en partant de l'air atmosphérique. De l'ensemble de toutes ces recherches de laboratoire, expériences à plus grande échelle et tentatives de fabrication, il résulte indubitablement aujourd'hui que la fixation de l'azote atmosphérique est un fait acquis, dont la réalisation industrielle va se poursuivre suivant deux voies parallèles: l'une représentéé par la fabrication du cyanamide-calcium, et l'autre par la combinaison directe de l'oxygène et de l'azote, sous l'influence d'arcs électriques de nature et de forme appropriées.

Les essais de MM. Franck et Carro, exécutés dansles laboratoires de la maison Siemens et Halske, ont abouti à des procédés pratiques, qui sont, depuis quelque temps, mis en exploitation par la «Cyanidgesellschaft " de Berlin, fondée par l'initiative de la Société Siemens et Halske et du « Gold und Silber Scheide Austadt " de Francfort. Une usine de 10.000 chevaux, située en Italie, à Piano d'Orte, vient d'être mise en marche pour cette fabrication du cyanamide-calcium; elle appartient à la «Société des Produits azotés " de Rome, concessionnaire des brevets Frank et Carro pour l'Italie et l'Autriche-Hongrie. Cette Société, qui est une filiale de la "Società Italiana dell' Elettrochimica», entreprend d'autre part la construction d'une seconde usiné Fiume, qui sera capable, nous assure-t-on, de fournir 12.500 tonnes de cyanamide par an. En France, on ne reste pas inactil, et si l'on ne peut encore signaler aucune usine de ce genre, il est à présumer qu'il s'en érigera sous peu. De toutes part, nous entendons parler de projets de eréation d'usines hydro-élertriques, ayant pour objet cette fabrication. Certains groupes financiers et industriels très puissants, que nous n'avons pas la permission de nommer, ont actuellement ell mains à peu près tous les éléments voulus pour entreprendre, avec succès, ta fabrication du cyanamide sur une grande échelle.

D'autre part, la publication des résultats obtenus par MM. Birkeland et Eyde avec leurs trois fours de 500 kilowats cha. cun, marchant, paraît-il, d'une façon régulière, depuis bientôt un an, à l'usine de Notodden en Suède, a mis en éveil le monde des affaires et suscité une légion d'inventeurs. Il n'est que bruit dans les milieux techniques, de nouveaux dispositifs comportant de notables (?) perfectionnements aux moyents d'augmenter la puissance efficiente des arcs électriques déviés 
dans le champ magnétique, de réduire encore leur effet disso. ciant, d'accroître les intensités et les tensions du courant générateur des arcs, en un mot, de multiplier le rendement et la capacité de production des appareils, tout en réduisant la dépense de leur entretien. Que va-t-il sortir de tant de fébrile activité? Sans aucun doute de nouveaux progrès dans cette fabrication. Mais sans les escompter, des usines peuvent déjà tabler sur les résultats actuels des appareils Birkeland et Eyde. Leur rendement est de 700 kilos d'acide nitrique commercial par kilowatt-an. Lacide produit par les fours en marche est transformé en nitrate de chaux pour l'agriculture, et titre en moyenne $13 \%$ d'azote, contre 14 à $16 \%$ qu'en contiennent les salpêtres du Chili. Le kilowatt-an étant estimé à 25 francs, les auteurs indiquent une dépense de 46 francs d'énergie électrique par tonne de nitrate. Comme les cours du nitrate du Chilli oscillent entre 270 et 290 francs la tonne, on voit qu'il y a place, entre le prix de revient et le prix de vente, a un joli bénétize déduction faite de toutes autres dépenses de maind'euvre, entretien et frais généraux.

C'est la «Norsk Elektrochemisch Selskab n fondée au capital de 7 millions de couronnes qui exploite ce procédé. Elle est formée de capitaux français, russes, danois et suédois. Le succès de cette première usine lui paraît réserver un avenir tel, dit-on, qu'on lui prête l'intention de créer une affaire colossale, au capital de 120 millions pour monter des usines partout sù l'on peut aménager des chutes d'eau à très bon marché. II nous revient qu'en attendant, cette "Société norvégienne de l'azote 》 a acquis près de Telemarken la chute de Svaelgfos, capable de fournir 25 ooo chevaux, pour les faire servir à cette fabrication dans une nouvelle usine. On songerait à ce qu'il paraît a capter des chutes de 200000 chevaux à cet effet !... Le champ des utilisations de forces hydrauliques ouvert par la fabrication électrochimique des engrais est vaste; n'a-t-on pas calculé qu'avec le rendement actuel du procédé Birkeland et Eyde, il faudrait employer 2 millions de chevaux pour produire une quantité de nitrate égale à celle exportée par les gisements du Chili.

Quoiqu'il en soit de ces projets d'entreprises monstres, il n'en résulte pas moins que la roie est franchement ouverte. Et comme nous le disions plus haut, les inventeurs de procédés, les vendeurs de chutes d'eau et les chercheurs de capitaux s'agitent fiévreusement autour de cette question de l'électrochimie des nitrates. Nous percevons les vibrations qui résultent de ce mouvement, par les demandes de renseignements techniques nombreuses et variées, qui nous sont adressées relativement aux créations de forces hydrauliques susceptibles de donner de grandes puissances à très bon marché. N'est-il pas des correspondants qui, de Paris, nous demandent à ce propos s'il est possible d'avoir des chutes où le cheval-an reviendrait à 10 francs aux bornes des génératrices !...

Nous ne voulons décourager personne, car nous avons nousmême la plus grande confiance en l'avenir de cette industrie des nitrates, qui est capable de faire la fortune de nos Alpes et de nos Pyrénées; mais nous exprimerons cependant l'avis que pour obtenir des résultats à même de rendre viables les entre. prises en état d'élaboration, il serait sage de voir (je parle pour chez nous), moins en grand. Ce n'est pas tout que de résoudre un problème au point de vue technique, il faut encore que sa solution satisfasse à certaines conditions commerciales hors desquelles il n'est point de bénéfices. Certes, le débouché des nitrates en Agriculture est, on peut le dire, illimité, d'autant plus qu'on peut prévoir à brève échéance l'épuisement des mines du Chili et que l'emploi de cet engrai se répandra plus à mesure de l'abaissement du prix de vente; mais toutes les questions relatives au prix de revient des nitrates électrochimiques, à leur composition et, par saite, à leur valeur culturale sont-elles résolues d'une manière tellement probante qu'on puisse, sans crainte, monter des usines de fabrication colossales? Nous croyons que les plus avisés sont ceux qui se proposent d'amorcer cette fabrication dans des usines modestes, considérées encore comme des usines d'essai, utilisant des chevaux résiduels qu'on peut trouver à très bon marché dans certaines de nos grandes chutes d'eau. Peu à peu, et sans doute assez vite, les appareils se perfectionneront, les rendements s'amélioreront, les électrochimistes feront l'apprentissage des meilleurs tours de mains donnant le meilleur produit, les prix de revient seront déterminés dans tous leurs éléments; parallèlement la valeur de l'engrai nouveau s'appréciera au moyen d'applications de plus en plus concluantes, et tout cela s'opèrera sans faire courir de risques aux capitaux engagés dans ces affaires. Nous craignons qu'en voulant au contraire bâtir de colossales entreprises financières et industriellcs sur la promesse des premiers résultats qui nous viennent de l'étranger, l'on ait à déplorer des insuccès retentissants, capables de retarder très longtemps l'essor de cette grande industrie. Des faits semblables qui ne datent pas de loin dans l'histoire de l'électrochimie et qu'il n'est pas besoin de nommer pour qu'on sache ce dont nous voulons parler, doivent servir d'exemples salutaires à ceux qui, ayant le désir de voir la jeune industrie grandir vite chez nous, sont portés à la faire périr d'indigestion.

Et pour terminer, nous dirons que nous croyons le jour peut-être pas très éloigné, où se réalisera cette idée transcrite ici par nous il y a déjà trois ans, et tout récemment brevetée paraît-il, par une puissante maison étrangère qui se propose de la mettre en pratique, idée consistant en ceci : réunir dans la même usine le four à synthèse de l'acide nitrique par action directe de l'arc électrique, et le four à cyanamide; le premier employant le mélange convenable d'oxygène et d'azote, livré par les appareils à liquéfaction partielle de l'air, et le second l'azote pur sous-produit de cette opération. Nous sommes ici dans le domaine des grands projets réalisables à brève échéance, et nous leur soubaitons bonne chance. E. F. Côte.

\section{Tachymètre bifluide}

Il existe de nombreux types de tachymètres basés sur l'action de la force centrifugue; malheureusement. ces appareils sont presque tous munis de ressorts qui, a la longue, se fatiguent et qui en outre ont besoin d'un dégraissage constant; l'appareil de MM. W.-E. Mouldsale et $C^{i e}$, de Liverpool, ne présente pas cet inconvénient, car il est basé sur la force centrifuge et la différence de densité de ceux fluides.

L'appareil, disposé verticalement, est constiué par une capacité de forme cylindrique $A$, qui communique par l'intermédiaire du tube $C$, avec un tube vertical $E$; à l'intérieur de ce tube $E$, se trouve un second tube $B$, ouvert à sa partie supérieure, qui communique au moyen des tubes recourbés avec l'intérieur de la capacité $A$.

Dans la capacité,' A est mis un fluide de grande densité, dont le niveau atteint la ligne pointillée; audessus est placé un liquide coloré moins dense qui ne $s \approx$ melange pas avec le fluide lourd; quand l'appareil est' au repos, le liquide est au même niveau dans le tube intérieur $\mathrm{B}$ et dans le tube $\mathrm{E}$ qui l'enveloppe Quand l'appareil tourne autour d'un axe vertical, sous l'action de la force centrifuge, le liquide dense est projeté vers l'extérieur, il fait monter le fluide léger dans les tubes $\mathrm{D}$, et par conséquent łans le tube $B$; en même temps, celui.ci baisse dans le tube E. Plus la vitesse de rotation est grande, plus le niveau du liquide est élevé dans le tube $B$; on peut donc, après"étalonnage,: lire la vitesse

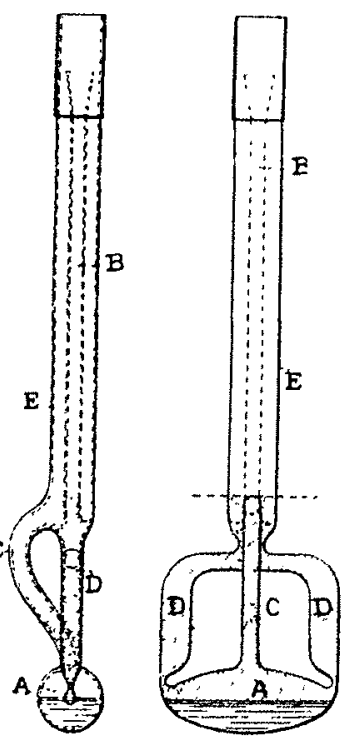
sur une graduation analogue à celle d'un thermomètre. 